\title{
Endoscopic optical coherence tomography with a flexible fiber bundle
}

Lara M. Wurster

Laurin Ginner

Abhishek Kumar

Matthias Salas

Andreas Wartak

Rainer A. Leitgeb 


\title{
Endoscopic optical coherence tomography with a flexible fiber bundle
}

\author{
Lara M. Wurster, ${ }^{\mathrm{a}, \star}$ Laurin Ginner, ${ }^{\mathrm{a}, \mathrm{b}}$ Abhishek Kumar, ${ }^{\mathrm{a}}$ Matthias Salas, ${ }^{\mathrm{a}, \mathrm{b}}$ Andreas Wartak, ${ }^{\mathrm{a}}$ and \\ Rainer A. Leitgeb ${ }^{\mathrm{a}, \mathrm{b}}$ \\ ${ }^{a}$ Medical University of Vienna, Center for Medical Physics and Biomedical Engineering, Vienna, Austria \\ ${ }^{b}$ Medical University of Vienna, Christian Doppler Laboratory for Innovative Optical Imaging and Its Translation to Medicine, Vienna, Austria
}

\begin{abstract}
We demonstrate in vivo endoscopic optical coherence tomography (OCT) imaging in the forward direction using a flexible fiber bundle (FB). In comparison to current conventional forward-looking probe schemes, our approach simplifies the endoscope design by avoiding the integration of any beam steering components in the distal probe end due to two-dimensional scanning of a focused light beam over the proximal FB surface. We describe the challenges that arise when OCT imaging with an FB is performed, such as multimoding or cross coupling. The performance of different FBs varying in parameters, such as numerical aperture, core size, core structure, and flexibility, was consequently compared, and image quality degrading artifacts were described in detail. Based on our findings, we propose an optimal FB design for endoscopic OCT imaging. (c) The Authors. Published by SPIE under a Creative Commons Attribution 3.0 Unported License. Distribution or reproduction of this work in whole or in part requires full attribution of the original publication, including its DOI. [DOI: 10.1117/1.JBO.23.6.066001]
\end{abstract}

Keywords: optical coherence tomography; endoscopy; fiber bundle.

Paper 180086RR received Feb. 8, 2018; accepted for publication May 24, 2018; published online Jun. 13, 2018.

\section{Introduction}

Optical coherence tomography (OCT) is a noninvasive imaging technique that performs cross-sectional imaging of transparent, translucent, or turbid media at a micrometer scale resolution. ${ }^{1}$ Being already well established in ophthalmology, ${ }^{2}$ OCT also holds the potential to become an important tool for in vivo imaging of internal organs by combination with endoscopy. To investigate less accessible tissues in the human body and tissues shielded by opaque media, an integration of the coherent imaging technique into endoscopic instruments proved successful. ${ }^{3,4}$ Cancer lesions and other types of pathologic tissue changes typically arise from close underneath the tissue surface, which is well accessible for OCT. Therefore, endoscopic OCT enables disease diagnosis in internal organs at an early stage and complements the information obtained by white-light endoscopy. ${ }^{5}$ In a best-case scenario, thus, endoscopic OCT avoids the need to remove tissue samples by providing morphological tissue information at up to $2 \mathrm{~mm}$ in depth and, therefore, also being considered as "optical biopsy." Furthermore, endoscopic OCT shows a tremendous impact in imaging of regions where common biopsy cannot be performed without introducing major risks, e.g., in the vascular system or in the brain. ${ }^{6}$

First implementations of OCT endoscopes aimed at imaging tubular organs (e.g., blood vessels) circumferentially, by directing the light beam sideways, perpendicular to the organ wall while performing a rotation and an axial translation of the miniaturized optics. ${ }^{4}$

Evidently, side imaging probes present severe limitations for imaging of hollow organs where the tissue of interest is located directly in front of the endoscope, such as the human bladder.

*Address all correspondence to: Lara M. Wurster, E-mail: lara.wurster@ meduniwien.ac.at
Forward imaging endoscopy probes have been developed to address this problem; however, in general a more sophisticated instrument housing a two-dimensional (2-D) scanning unit is required. Here, the most commonly used mechanisms integrate either a microelectromechanical system scanner or a piezo tube. $^{7-9}$ The former, however, requires a folding of the beam path, consequently increasing the overall size oft the instrument. On the other hand, the piezo tube solution requires a sophisticated image reconstruction algorithm due to a spiral scan pattern, which, in addition, is sampled nonuniformly. Previous reports have shown that the integration of a 2-D scanning mechanism in a fairly small endoscope seems to be the major challenge for the development of a forward imaging probe. Furthermore, optical and biocompatibility requirements need to be considered and add complexity to the design for all the aforementioned endoscopic imaging approaches. ${ }^{10}$

An alternative endoscope design capable of imaging in the forward direction and avoiding scanning at the distal end employs a different component-a fiber bundle (FB). Here, scanning of the light beam can be performed at the proximal end, using a pair of galvanometer mirror scanners ${ }^{11}$ or a lens system with stage control ${ }^{12}$, hence avoiding the need of any moving parts to be integrated into the endoscope head. Focusing of the light at the distal end of the FB can be performed with a small GRIN lens or a lens system. ${ }^{11,12}$

It is important to note that individual fibers of a coherent FB in general exhibit different optical properties in comparison to a standard single-mode (SM) fiber. Various factors, such as core size, package density, and numerical aperture (NA), strongly influence the transmission behavior and need to be carefully taken into account. Associated effects, such as multimoding within each core, cross coupling (or cross talk) of the light among multiple cores, or coupling and transmission losses, might severely degrade image quality, ${ }^{13,14}$ especially of the 
OCT tomogram. ${ }^{11,12,15-19}$ Multimoding becomes mostly prominent in FBs of high NA (i.e., large difference in refractive index between core and cladding) and/or large core size and may result in axial ghost artifacts within the OCT tomograms. ${ }^{12,15}$ FBs exhibiting multimodal behavior are, however, less likely to cross couple the light since they are able to better confine light to their respective cores due to the mentioned refractive index difference. Cross coupling, therefore, typically arises when the NA is small and the cores are closely packed. ${ }^{13}$ As a consequence of both, multimoding and cross coupling, the OCT detection sensitivity is reduced by a loss in modulation contrast across the detected interference pattern as described by Risi et al. ${ }^{16}$ Other image quality degrading effects specific to FB imaging (but independent of its NA) are the pixelation artifact as well as reflections at the front and back surface of the FB. The latter can be avoided by angle polishing at both ends and, therefore, preventing backreflected light from the distal end to be coupled back into the core or from the proximal end to obscure the detected signal. In a different, simpler OCT imaging approach, the reference and sample arm share the same optical pathway. In this so-called common-path OCT configuration, the backreflection of light at the distal surface of the endoscope serves as the reference plane or an additional reference surface is added distally. ${ }^{11,16}$ However, such a configuration restricts the use of means for detection with optimal sensitivity by excluding balanced detection (BD). The latter is indispensable in current swept-source OCT to maintain sensitivity close to the shot noise limit at high imaging speeds. On the other hand, the pixelation artifact can only be removed in a postprocessing step by averaging of multiple images or by the application of filtering. ${ }^{18,20}$ Another shortcoming of previously reported FB endoscopes regards their limitation in terms of flexibility. The use of rigid FBs severely restricts their application for imaging of hollow organs in vivo but imaging of ex vivo tissue samples was reported. ${ }^{12}$ However, the presented image suffered from ghost artifacts due to the multimodal behavior of the FB, although a relatively long wavelength of $1310 \mathrm{~nm}$ was used. Previous OCT investigations using flexible FBs reported imaging of nonorganic phantoms. ${ }^{16,19}$ So far, none of the published works on rigid or flexible FBs reported in vivo results.

In this paper, we present a custom-developed flexible FB forward imaging OCT endoscope. Our aim is to investigate flexible FBs and in particular FBs where the image is not affected by ghost artifacts due to multimoding. We compare the performance of two commercially available flexible FBs in addition to the rigid one used by Ref. 12 . We finally selected the better suited flexible FB to demonstrate the capabilities of our developed FB OCT endoscope by presenting, to the best of our knowledge, first in vivo imaging results. On the one hand, image degradation due to cross coupling between cores, and, on the other hand, improper coupling into the cores are investigated in detail. Based on our findings, an optimal FB design for endoscopic OCT imaging is eventually proposed.

\section{Methods}

A schematic drawing of the setup is shown in Fig. 1. A commercially available tunable laser source (TLS) centered at a wavelength of $1040 \mathrm{~nm}$ with a bandwidth of $100 \mathrm{~nm}$ and a sweep rate of $100 \mathrm{kHz}$ (Axsun Technologies, Inc.: 1060 Swept Laser Engine) was employed, and its integrated $k$-clock was used to avoid rescaling of the acquired spectra in postprocessing.

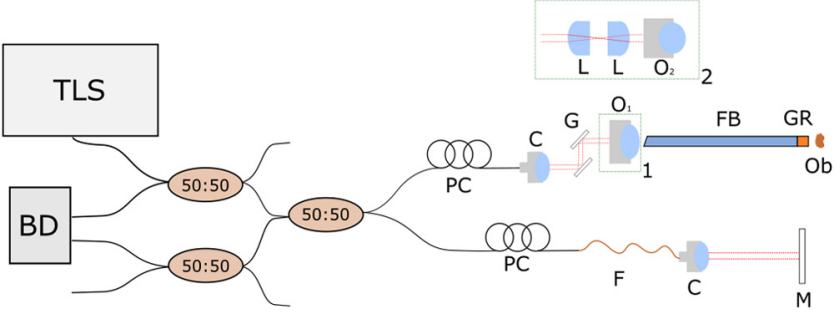

Fig. 1 Scheme of the OCT setup to perform imaging with the customdeveloped FB forward imaging OCT endoscope: TLS, tunable light source; $\mathrm{BD}$, balanced amplified photodetection unit; $\mathrm{PC}$, polarization control paddles; $\mathrm{C}$, fiber collimator; $\mathrm{G}$, galvo scanner; $\mathrm{O}_{1}$, objective (Thorlabs scan lens); $\mathrm{O}_{2}$, objective (Nikon); Ob, object; FB, fiber bundle; M, mirror; F, SM fiber; GR, GRIN lens; L, lens, and boxes 1 and 2, different beam focusing schemes (depending on respective FB).

The theoretical axial resolution of the system was determined to be $4.8 \mu \mathrm{m}$ (in air), and a sensitivity of $97.8 \mathrm{~dB}$ was measured close to the zero delay with a mirror as a sample and without the FB in the sample arm. The low coherent light emitted by the TLS was guided through a fiber-based symmetrical Michelson interferometer, ${ }^{21}$ which consisted of three $2 \times 2$ fiber couplers (Thorlabs, Inc.: $2 \times 2$ Wideband Fiber Optic Coupler) at a splitting ratio with regards to optical power of 50/50 each. In the sample arm, the light was collimated to a beam of $3.37 \mathrm{~mm}$ in diameter using a fiber collimator (C; Thorlabs, Inc.: F260APC$1064, f=15.43 \mathrm{~mm}$; NA $=0.16)$. The collimated beam was directed by a pair of galvo mirrors (G; Thorlabs Inc.: GVS002) to a scan lens (Fig. 1, box 1) or scanning lens system (Fig. 1, box 2) to achieve the best possible combination of NA and spot size fitting the respective FB's NA and core size. The scan lens $\left(\mathrm{O}_{1}\right.$; Thorlabs Inc.: LSM02-BB) enabled telecentric scanning of the laser beam across the proximal end of the FB (FB3, Table 1) without the need of any additional lenses. For a different FB (FB2, Table 1), a smaller spot size was necessary, and a high NA objective $\left(\mathrm{O}_{2}\right.$; Nikon: $40 \times / 0.65$, WD 0.65$)$ was chosen in combination with a $4 f$ lens configuration (L; Thorlabs Inc.: AC254-040-B) in front of the objective to again enable telecentric scanning of the focused light beam. At the distal end of the FB, the light was focused by a GRIN lens (GRINTech: GT-LFRL-180-cust-50-NC) at a working distance of $\sim 670 \mu \mathrm{m}$ with a 1:1 magnification and a size similar to the diameter of

Table 1 Overview of FB specifications.

\begin{tabular}{lccc}
\hline Specifications & FB1 & FB2 & FB3 \\
\hline Company, name & $\begin{array}{c}\text { Schott Image } \\
\text { Conduit }\end{array}$ & $\begin{array}{c}\text { Fujikura } \\
\text { FIGH-40-920G }\end{array}$ & $\begin{array}{c}\text { Fujikura } \\
\text { FIGR-10 }\end{array}$ \\
Number of fiber cores & 50,419 & 40,000 & 10,000 \\
Diameter $(\mathrm{mm})$ & 3.2 & 1.03 & 1.5 \\
Length $(\mathrm{cm})$ & 30 & 30 & 30 \\
Bending radius $(\mathrm{mm})$ & Rigid & 100 & 300 \\
Core diameter $(\mu \mathrm{m})$ & 9 & $\sim 3$ (irregular) & 6.1 \\
NA & 0.55 & 0.35 & 0.12 \\
Cutoff wavelength $(\mu \mathrm{m})$ & 6.5 & 1.37 & 0.96 \\
\hline
\end{tabular}


the FB itself. The resulting lateral field-of-view (FOV) is, therefore, equal to the FB diameter with a theoretical lateral resolution of twice the core spacing of the respective FB. The proximal end of each FB was polished at an angle of 8 deg to avoid any back coupling of reflected light into the detection unit. Back reflections at the distal end of the FB are reduced by the attachment of the GRIN lens. The GRIN lens itself at the distal end was not angle polished (cf. Fig. 1). Consequently, light reflected from the GRIN lens surface contributed to the detected interference signal but was not obstructing the sample image features since the position of the zero delay was chosen to avoid any overlap. To compensate for dispersion mismatch introduced by the respective $\mathrm{FB}$, additional length-matched SM fibers were inserted into the reference arm. The interference pattern was finally recorded by a dual-BD unit (Thorlabs Inc.: PDB130C) at a frequency of up to $350 \mathrm{MHz}$ at 12-bit resolution via a PCIe DAQ board (AlazarTech, Inc.: ATS9360).

Three FBs differing in terms of their respective hardware specifications (cf. Table 1) were compared for applicability to perform endoscopic OCT investigations.

To estimate the properties of a fiber in terms of the number of modes that are traveling through the core, the normalized wavenumber $(V)$ can be determined by the following equation:

$V=\frac{\pi \cdot d \cdot N A}{\lambda}$,

where $d$ denotes the diameter of the core of the individual fiber. A $V$ number $>2.405$ by definition no longer supports SM behavior. $^{22}$

Using Eq. (1), the cutoff wavelength, which determines the maximum wavelength still supporting SM behavior, for each of the respective FBs was calculated and is stated in Table 1. Since the used light source is centered at a wavelength of $1040 \mathrm{~nm}$, SM behavior was only expected for FB3 (due to its low NA).

As mentioned earlier, the lens configuration to couple light into the individual cores of the FB was chosen to match the core size and NA of the FB. Consequently, for experiments conducted with FB2, the scheme described in Fig. 1 box 2 was used providing a spot size and NA of $\sim 1.7 \mu \mathrm{m}$ and 0.37 , respectively. For FB3, a larger spot size but smaller NA was better suited to couple light efficiently into each core. Thus, the scheme in Fig. 1 box 1 provided a spot size and NA of $7.0 \mu \mathrm{m}$ and 0.09 , respectively.

\section{Results}

\subsection{Detailed Investigation of OCT-Related FB Artifacts}

In general, OCT performed through FBs suffers from three artifacts: multimoding, group delay differences between fiber cores, and cross coupling between cores. The aforementioned pixilation artifact is common to all FBs and not specific for OCT. Additionally, light is not always efficiently coupled into the FB cores during scanning across the FB facet. In the following, we describe the above-mentioned effects individually for each of the three FBs listed in Table 1.

\subsubsection{Multimoding}

Multimoding within fibers results in axially displaced ghost images of the structures ${ }^{12,15}$ in OCT tomograms that might severely overlap with the original structure terms and, furthermore, reduces modulation contrast and consequently sensitivity. ${ }^{16}$ As expected, according to the analysis following Eq. (1), OCT imaging performed with FB1 suffers significantly from multimoding artifacts. Only above a wavelength of $6.5 \mu \mathrm{m}, \mathrm{SM}$ behavior is guaranteed (Table 1). In addition, a relatively large separation between individual fibers $(6 \mu \mathrm{m})$ results in a prominent pixelation artifact in cross sectional as well as en face images. Still, successful OCT imaging has previously been demonstrated by Xie et al. ${ }^{12}$ using the same but longer FB in combination with a $1310-\mathrm{nm}$ wavelength light source. However, even for the employed longer FB, one ghost image remained within the imaging range. Given the strong multimoding at $1060 \mathrm{~nm}$ as well as the fact that the FB is rigid, we discarded FB1 from the subsequent investigations.

Artifacts due to multimoding are not expected in FB3, due to the very small NA, resulting, as mentioned earlier, in a low cutoff wavelength of $960 \mathrm{~nm}$. Consequently, only an SM contributes to the detected signal. Also for FB2, multiple modes cannot be observed in the cross-sectional images, although a cutoff wavelength of $1.37 \mu \mathrm{m}$ was determined. This fact can be attributed to the FB's length that shifts additional modes out of the imaging range. This assumption is confirmed by previously published results (cf. Ref. 15) where a large mode separation (4.4 $\mathrm{mm}$ for $0.8-\mu \mathrm{m}$ wavelength) for an FB with a length of $30 \mathrm{~cm}$ was estimated.

\subsubsection{Group delay variations}

Another complication that can occur when imaging with an FB is performed is caused by group delay variations. The group delay defines the axial position of the measured structure within an OCT A-scan. Variations are mainly introduced by an irregular core size, as in FB2. Nevertheless, also cross coupling with light from neighboring cores, when imaging with an FB of very low NA (as for FB3) is performed, may introduce minor axial shifts. To further investigate the influence of difference in core size and NA on OCT tomograms, a more detailed examination was performed and is described in the following.

In an ideal scenario, a cross-sectional OCT image of a flat surface results in a line with the maximum intensity pixel in each A-scan at the same axial position. This is, however, not the case when imaging with an FB is performed. To visualize these effects, a mirror surface was imaged with both flexible FBs. After reconstruction of the OCT tomograms, a graph plotting the maximum intensity position of each A-scan within the respective tomograms with respect to a linear fit-corresponding to the theoretically flat mirror surface-is generated (cf. Fig. 2). In Fig. 2(a), the effect of the irregular core size of FB2 can be observed through large deviations of the individual intensity maxima from the linear fit. The graph for FB3 [Fig. 2(b)] shows much smaller deviation from the linear fit. The remaining axial offsets of the intensity maxima can be attributed either to cross coupling, ineffective coupling, or a combination of both. Figure 2 shows an exemplary single B-scan of FB2 and FB3, respectively. Taking 20 consecutive B-scans into account, the following results in terms of the deviation of the linear fit are obtained: FB2: mean deviation of $196.4 \mu \mathrm{m}$ (standard deviation $167 \mu \mathrm{m}$ ) and FB3: mean deviation of $23 \mu \mathrm{m}$ (standard deviation $23.5 \mu \mathrm{m}$ ). These results confirm that the difference of each core in terms of shape and size in FB2 degrades the integrity of OCT images; light travels a slightly different path length in each core and, consequently, introducing axial shifts in the A-scan. In theory, the referred 

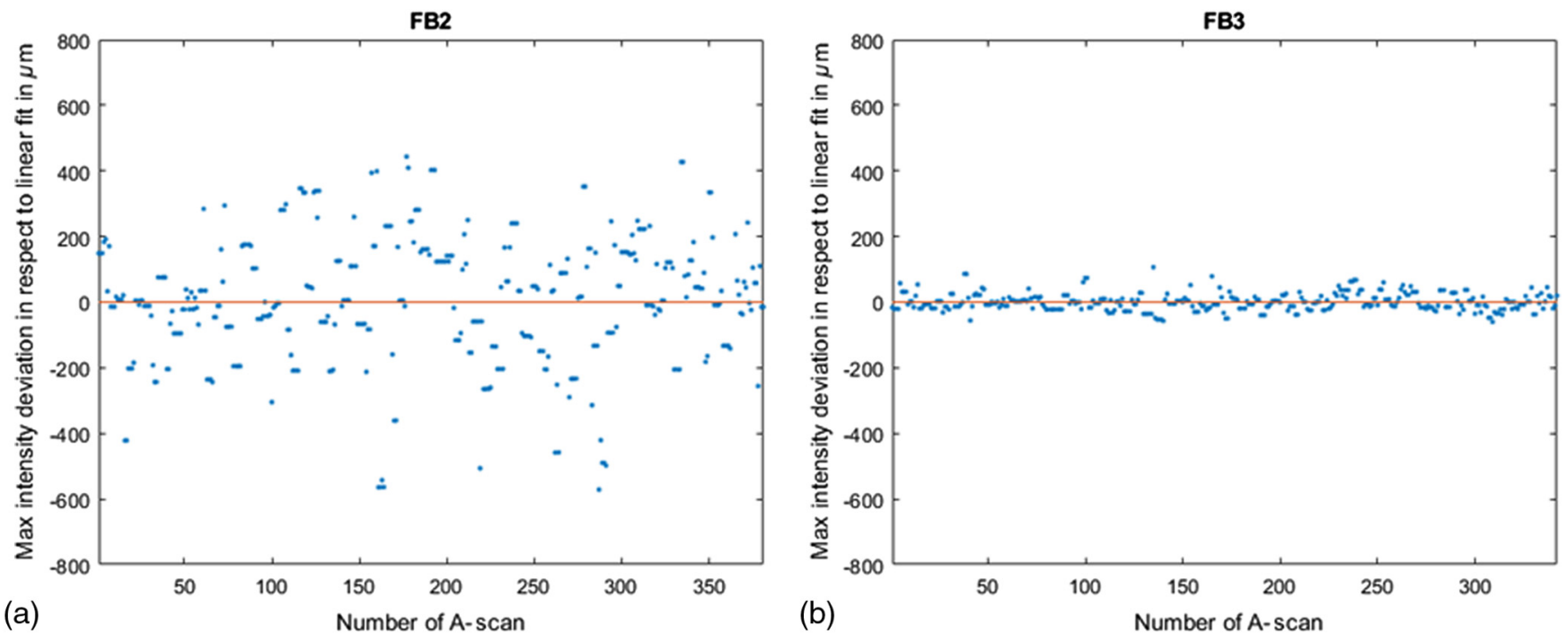

Fig. 2 Performance of FB2 and FB3 by plotting the difference in axial location of the maximum intensity position along a cross-sectional image of a flat surface (mirror): (a) result for a single B-scan acquired with FB2 and (b) result for a single B-scan acquired with FB3.

shifts could be corrected for in postprocessing by imaging a reference sample (i.e., flat mirror) in a calibration step and later applying the measured shifts to the final sample image. However, in practice, this calibration technique suffered from imprecision in repositioning of the galvo scanners and small mechanical fluctuations of the FB position relative to the scanners. This, again, resulted in inconsistent coupling into the cores of the FB, ultimately leading to remaining varying axial shifts. An alternative method is to use the reflection from the flat GRIN lens surface at the distal end that produces an OCT signal above the actual structure terms. Such calibration works fine for FB3, where the fluctuations remain small [cf. Fig. 2(b)]. For FB2, the fluctuations for the GRIN lens reflex only consume already $>1 \mathrm{~mm}$ of the depth range according to Fig. 2(a). With the axial structure extension consuming typically $\sim 2 \mathrm{~mm}$ of depth range, the necessary axial range adds rapidly up to more than $5 \mathrm{~mm}$, thus exceeding our and most FDOCT ranging settings. A common-path configuration using the distal end of the GRIN lens as reference surface would possibly attenuate the effect of group delay variations but comes with a loss in sensitivity due to the limited flexibility in controlling the reference arm power and lack of $\mathrm{BD}$, as mentioned earlier. As demonstrated in Fig. 2(b), characteristics of FB3 introduce a much smaller shift in each A-scan and can, therefore, be potentially used for OCT imaging.

\subsubsection{Cross coupling}

To investigate the effect of cross coupling on OCT images, the performance of FB2 and FB3 when imaging a resolution target (RT) is compared (Thorlabs Resolution Target R2L2S1P, 29 cycles $/ \mathrm{mm}$ ). To do so, en face mean intensity projections of the recorded volumetric data sets are generated. In Fig. 3(a), the individual cores of FB2 are visible, and the number and bars are clearly defined. This leads to the assumption that cross coupling is significantly reduced in FB2, due to the higher NA and, therefore, higher difference in refractive index of core to cladding. It should be noted that the FOV was limited by the objective at the scanning side to $\sim 500 \mu \mathrm{m}$, and the best resolution is only obtained in the central region of the image due to the small depth of field and angled proximal surface of the FB. On the other hand, the en face projection acquired using FB3 shows a degradation of image quality [cf. Fig. 3(b)]. The same number and bars of the RT as shown in Fig. 3(a) are imaged with a larger FOV and the individual cores are clearly visible. However, the bars and numbers of the RT are hardly identifiable. Consequently, it can be assumed that cross coupling takes place and does not only influence the axial information of a cross-sectional image [Fig. 2(b)] but also the en face information of a three-dimensional (3-D) OCT volume. The information is smeared out due to the light not being confined to a single core but transmitted also to neighboring cores. Furthermore, it can be observed that light is not equally coupled into each individual core and in some regions no light seems to be transmitted through the FB at all, possibly due to contaminated fiber facets.

Based on the above results, we conclude that imaging with FB2 can only be performed at a rather restricted performance due to the irregular core size being very critical in terms of OCT imaging. Although FB3 showed a sever degradation in image quality in the en face image, we conclude that OCT can still be performed since axial shifts of the signal, caused by cross coupling, were limited to a very small region. Consequently, further experiments and OCT measurements were conducted with FB3.

To examine the effect of cross coupling and imperfect positioning of the proximal scanning beam with regards to the center of one individual core in more detail, additional experiments were performed; Fig. 4(a) shows a peak obtained by optimizing the position of the focused beam with respect to the center of one individual core (blue curve) while illuminating a mirror in the sample arm. Therefore, the FB was slightly moved transversally to achieve a steep and narrow peak in the OCT A-scan. For this optimal coupling, an axial resolution of $\sim 8 \mu \mathrm{m}$ was determined. The difference to the theoretical resolution of $4.8 \mu \mathrm{m}$ is attributed to spurious cross coupling and dispersion mismatch. Thereupon, the FB was slightly moved in lateral direction resulting in the disappearance of the main peak. In this configuration, multiple smaller peaks appeared in the OCT A-scan 

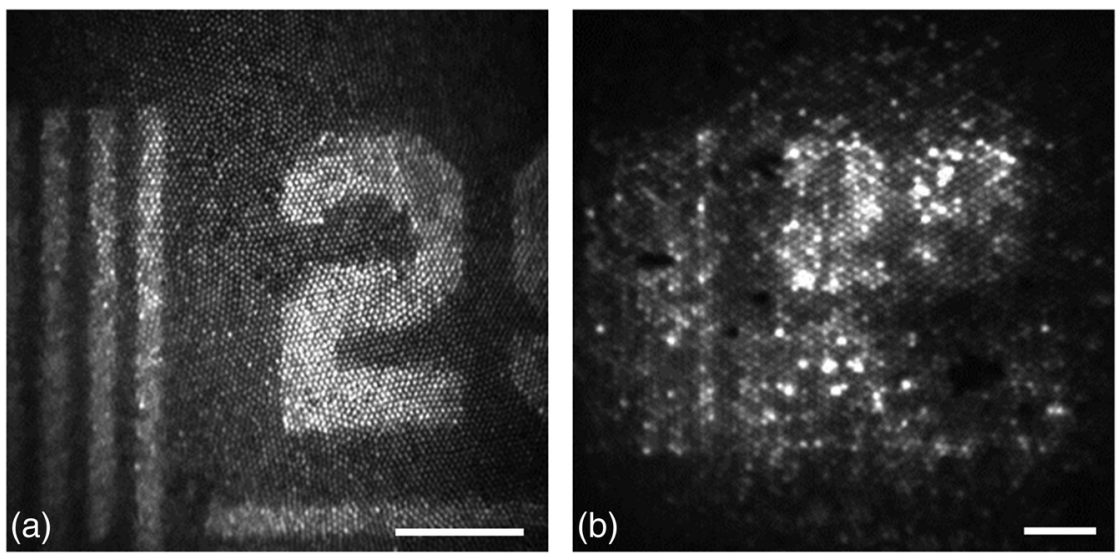

Fig. 3 En face projection of an RT imaged with FB2 and FB3 to compare their performance: (a) en face projection acquired with FB2 and (b) en face projection acquired with FB3. Scale bars: $100 \mu \mathrm{m}$.
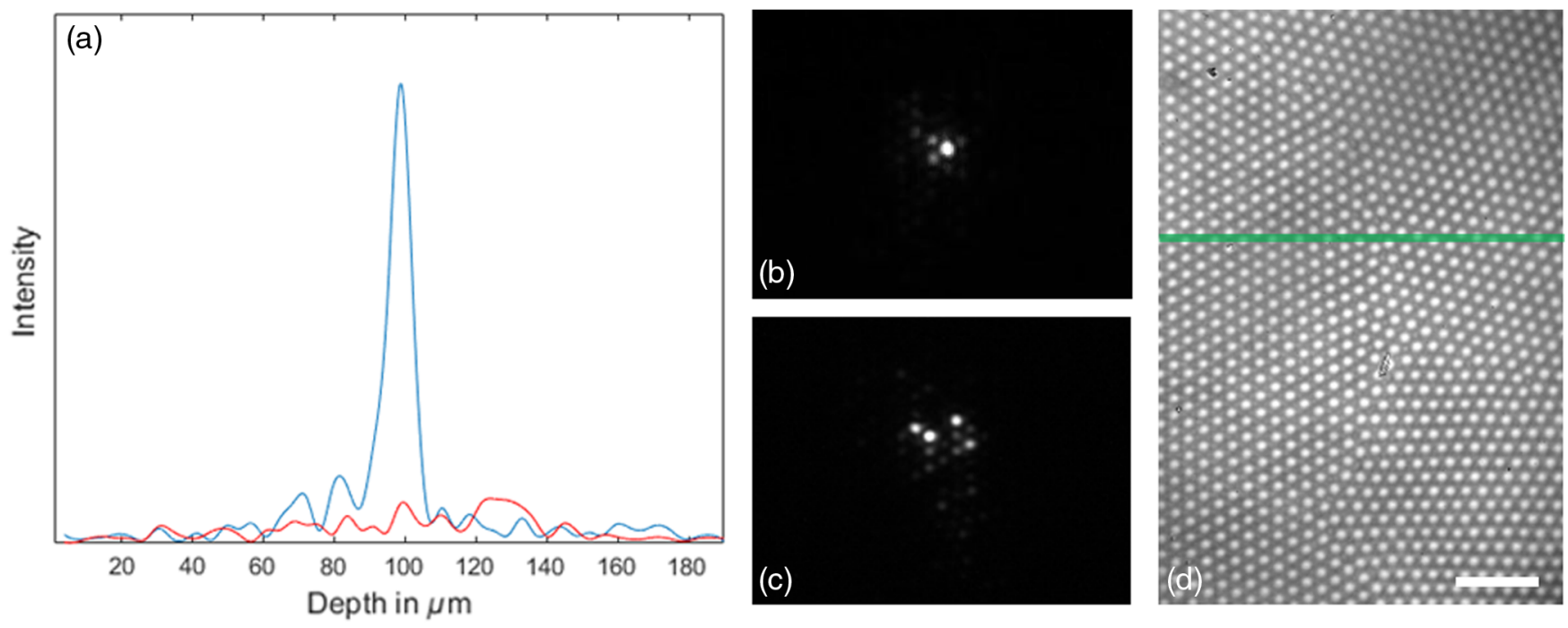

Fig. 4 Illustration of the coupling problem when using FB3. (a) Graph obtained by placing a mirror at the sample position and carefully moving the FB to obtain a narrow peak (blue) (indicating that light hits the center of an FB's core) and multiple smaller peaks (red) when moving the FB slightly laterally. (b) Image of the output of the FB when most of the light is coupled into a single core [corresponding to the blue curve in (a)] and (c) multiple cores [corresponding to the red curve in (a)]. (d) Image of the FB's facet to show the variety in arrangement of the fibers and indicating (green line) a scanning beam for an OCT B-scan. Scale bar: $50 \mu \mathrm{m}$.

[cf. Fig. 4(a), red curve]. After this measurement was performed, the mirror in the sample arm was replaced by two lenses (Thorlabs Inc.: AC254-030-B and AC254-200-B) to image and magnify the output of the FB onto a 2-D CMOS camera (Photonfocus, MV1-D2080-160-CL, $2080 \times 2080$ pixel resolution, $8 \mu \mathrm{m} \times 8 \mu \mathrm{m}$ pixel size). Using this setup, Figs. 4(b) $-4(\mathrm{~d})$ were obtained. Figures 4(b) and 4(c) were generated using the OCT light source, comparing the following two scenarios: (i) aiming the OCT beam directly at the center of one core [cf. Fig. 4(b)] and (ii) aiming the OCT beam not at a single core but at a region between multiple cores [cf. Fig. 4(c)]. The latter is further referred to as extrinsic cross coupling. Obviously, even light, which is perfectly coupled into a single core, is emitted by several cores at the distal end. These results confirm that intrinsic cross coupling does take place. Furthermore, this has an impact on the achievable lateral resolution. The theoretical lateral resolution stated in Sec. 2 holds only for a single core for illumination and detection. Even in the ideal case of Fig. 4(b), light from neighboring cores contributes to the lateral PSF and, thus, degrading the resolution. Taking Figs. 4(b) and 4(c) into account, it can be estimated that in the best case light is spread over \pm 1 cores or in the worst case \pm 2 cores. When taking Fig. 4(d) also into account, a core spacing of $\sim 12 \mu \mathrm{m}$ can be measured resulting in a theoretical resolution of $\sim 24 \mu \mathrm{m}$. Considering the light spreading over several cores, a degraded resolution of 6 times the core spacing (resolution of $72 \mu \mathrm{m}$ ) or 10 times (resolution of $120 \mu \mathrm{m})$ can be predicted. It can, therefore, be estimated that the lateral resolution is degraded from $\sim 24$ to 72 to $120 \mu \mathrm{m}$. Additionally, in a realistic scenario, the scanning beam is moved across the FB as indicated in Fig. 4(d). This image of the facet was obtained, by shining white light into the proximal end of the FB. The green line indicates the scanning beam to generate an OCT B-scan. It becomes immediately obvious 


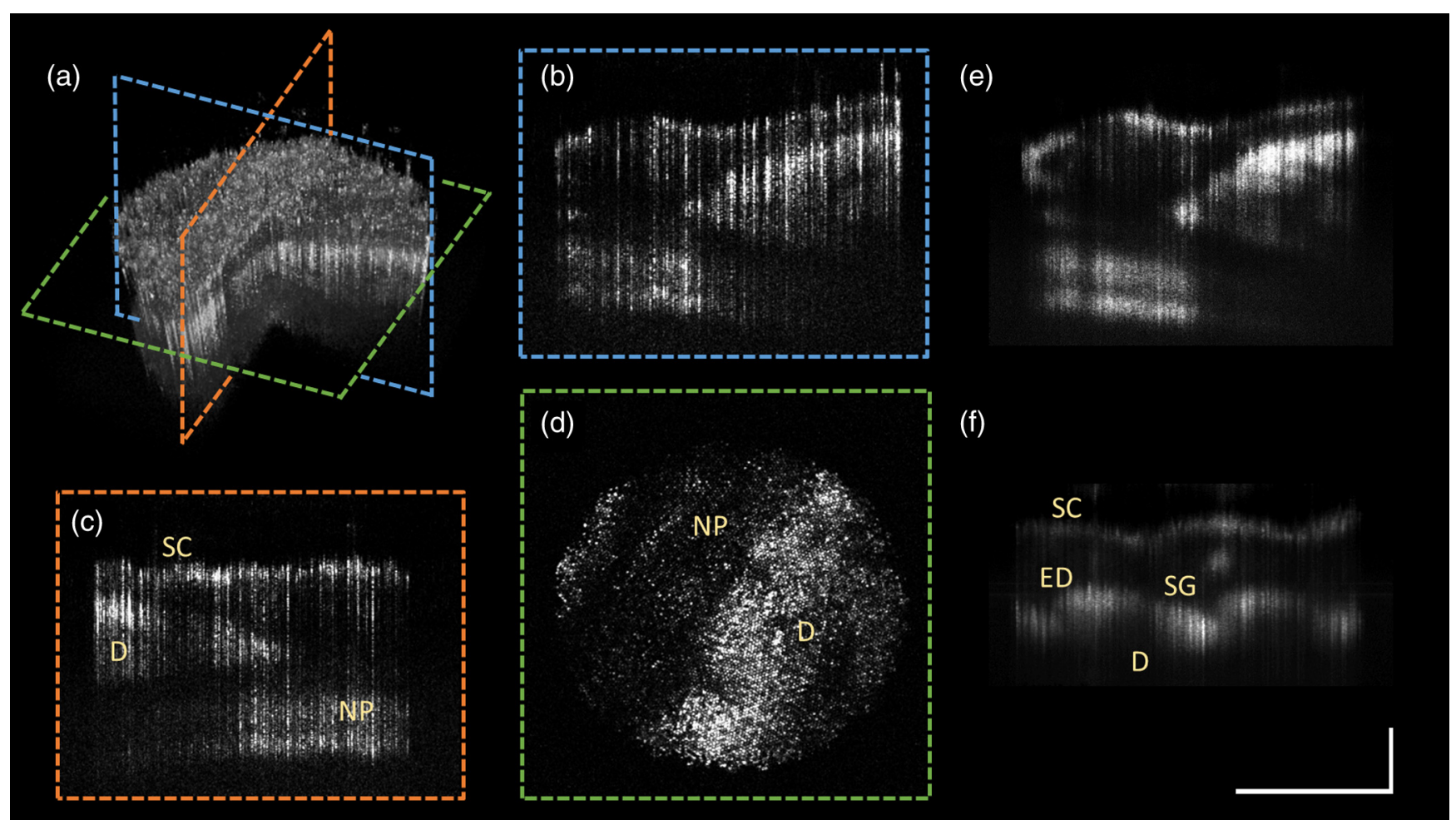

Fig. 5 In vivo imaging results of the nail bed of (a)-(e) a human finger and (f) finger pad. (a) 3-D representation of the nail bed, (c) and (d) single frames from positions indicated by the colored boxes in (a), (e) 40 times averaged image of consecutive cross-sectional images of the nail bed, and (f) 250 times averaged image of a human finger where a sweat gland can be identified. SC, stratum corneum; D, dermis; NP, nail plate; ED, epidermis; and SG, sweat gland. Scale bars $500 \mu \mathrm{m}$ for all images.

that the scanned light beam does not always coincide with the central position of a core, resulting in extrinsic cross coupling and severe degradation of the OCT signal as well as the lateral resolution in such cases. Furthermore, Fig. 4(d) shows that fibers are in general arranged linearly but the orientation of these lines obviously varies across the FB. Therefore, an alignment of the FB's core structure with respect to the scanning beam implicates a fundamental challenge.

\subsection{In Vivo Measurements}

Figure 5 shows images of a human finger nail bed and finger pad acquired with FB3. Informed consent from the healthy volunteer was obtained prior to the measurements, and the study was in agreement with the tenets of the Declaration of Helsinki and approved by the institutional ethics committee. One acquired 3-D-stack contains $700 \mathrm{~B}$-scans with $700 \mathrm{~A}$-scans each and 680 depth pixels. A sensitivity of $86 \mathrm{~dB}$ was measured (when carefully coupled into the center of a single core of the FB). Gel was applied onto the sample for reasons of refractive index matching and, thus, increasing the penetration depth by decreasing specular reflections at the air/tissue interface. To compensate for the axial shifts in each A-scan, a calibration with the flat GRIN lens surface was applied to all images as mentioned in Sec. 3.1.2.

Figures 5(a)-5(e) show exemplary representations of the finger nail bed. A rendered 3-D visualization is shown in Fig. 5(a), and single frames from positions indicated by the colored frames in (a) are shown in Figs. 5(b)-5(d). Figure 5(e) shows an average projection of 40 consecutive scans in the proximity of the tomogram as shown in Fig. 5(b). Averaging of multiple B-scans acquired at the exact same location does not necessarily improve the contrast in an FB-based system, since it will only enhance the core structure. However, by moving one axis of the galvo scanner over a small region only, while keeping the deflection of the other axis, averaging over multiple cores can be performed. An average of 250 images of a finger pad is shown in Fig. 5(f).

Obviously, if compared to images acquired with a standard bench-top OCT system, the images presented in Fig. 5 suffer from aforementioned shortcomings that the use of an FB for endoscopic OCT imaging brings along (cf. Sec. 3.1). However, morphological structures, such as the nail plate, dermis, epidermis, and sweat glands, of the human finger can be identified.

\section{Discussion}

In this paper, we presented first in vivo OCT imaging results obtained with a flexible FB where scanning was performed at the proximal end. This has the advantage that any beam scanning device can be avoided at the distal end and imaging in the forward direction is feasible. The presented approach implicates image degrading shortcomings related to well-known characteristics of FBs, such as cross coupling or multimoding. Experiments were conducted with three different FBs. However, FB1 was quickly excluded from further experiments after confirmation was obtained that the multiple modes traveling through this bundle at the 1040-nm wavelength range would superimpose the image information. Nevertheless, we want to refer to Xie et al. ${ }^{12}$ who imaged ex vivo tissue using the 
same FB but at a longer wavelength $(1310 \mathrm{~nm})$ with only one remaining ghost image. Investigations with different phantom measurements using FB2 showed that the variation in core size is a great challenge for OCT. One possibility to eliminate the group delay variation is the use of an OCT common-path setup as demonstrated by several groups. ${ }^{11,16}$ The loss in sensitivity due to the missing $\mathrm{BD}$ and rigid reference arm power setting might, however, be critical. Although FB2 cannot be applied for OCT imaging, it has successfully been used in combination with a coherent anti-Stokes Raman scattering setup to image rabbit aorta $e x$ vivo. ${ }^{23}$ FB3 has a very low NA and, consequently, suffers from cross coupling that becomes mostly prominent when looking at an en face projection image, e.g., from an RT. Less prominent but still visible is the effect when looking at a cross-sectional image where it causes the detected signal to have a slight shift for each A-scan in the axial direction, and furthermore, a decline of the axial resolution is observed. Additionally, the axial resolution and shape of the signal in an A-scan depend on the position of the scanned beam in respect to the FB core. One option to avoid that problem is to use a reference image as shown in Fig. 4(d) where the individual cores are visible and, consequently, create a map to control the driving signals of the galvo scanners in a way that addresses each central point of a core individually.

Even though all the three applied FBs showed severe limitations in one or the other way, first in vivo imaging results could be obtained using FB3. Additionally, our observations allow the conclusion that the ideal FB for endoscopic OCT imaging would need particular specifications in terms of core size and NA to prohibit either multimoding or cross coupling of the transmitted light. It can be estimated that, when taking an NA of 0.35 and a central wavelength of $1040 \mathrm{~nm}$ into account, a very small but regular core size of roughly $\sim 2.3 \mu \mathrm{m}$ would be necessary to fulfill the above requirements. When using a longer wavelength, such as $1310 \mathrm{~nm}$, a slightly larger core size of $\sim 3 \mu \mathrm{m}$ would also be acceptable. However, to the best of our knowledge, such an FB is currently not available in the market. In theory, it would enable endoscopic OCT imaging at an improved performance with an estimated increase in sensitivity of $\sim 10 \mathrm{~dB}$, considering a behavior comparable to an OCT endoscope using an SM fiber. Such an endoscope would then be comparable to other forward imaging OCT endoscopes in terms of imaging quality, providing further advantages in terms of reduced complexity and lower costs. However, image quality would still be affected by the pixilation artifact, which is an intrinsic drawback when using an FB. Also, the proximal scanning would need to be controlled carefully to maintain optimal coupling conditions across the FOV.

\section{Conclusion}

In this work, we presented a flexible forward imaging FB endoscope for OCT at 1040-nm central wavelength. We identified current limitations that arise when coherent imaging with an FB is conducted and compared the performance of three different FBs. In addition to ex vivo phantom measurements, we present 2-D and 3-D in vivo imaging results of a human finger. Since a theoretically ideal FB for OCT imaging is currently not available in the market, the required specifications of such an FB to achieve improved imaging performance were proposed.

\section{Disclosures}

The authors declare that there are no conflicts of interest related to this article.

\section{Acknowledgments}

The authors acknowledge financial support from the European Union project MIB (Horizon2020, Contract No. 667933) and the Austrian Federal Ministry for Digital and Economic Affairs and the National Foundation for Research, Technology and Development. Furthermore, we would like to thank Bernhard Messerschmidt from GRINTECH GmbH, Jena, Germany, for providing us with GRIN lenses and Sebastian Dochow and Iwan Schie for their help and supply of FBs. Furthermore, we want to thank Marco Augustin, Bernhard Baumann, Danielle J. Harper, Pablo Eugui, Antonia Lichtenegger, Fabian Placzek, Elisabet Rank, Mikael Erkkilä, and Florian Beer for helpful discussions and assistance.

\section{References}

1. D. Huang et al., "Optical coherence tomography," Science 254, 11781181 (1991).

2. W. Drexler and J. G. Fujimoto, "State-of-the-art retinal optical coherence tomography," Prog. Retinal Eye Res. 27(1), 45-88 (2008).

3. G. J. Tearney et al., "In vivo endoscopic optical biopsy with optical coherence tomography," Science 276(5321), 2037-2039 (1997).

4. G. J. Tearney et al., "Scanning single-mode fiber optic catheter-endoscope for optical coherence tomography," Opt. Lett. 21(7), 543-545 (1996).

5. J. G. Fujimoto et al., "Optical biopsy and imaging using optical coherence tomography," Nat. Med. 1(9), 970-972 (1995).

6. M. J. Gora et al., "Endoscopic optical coherence tomography: technologies and clinical applications [Invited]," Biomed. Opt. Express 8(5), 2405-2444 (2017)

7. S. A. Boppart et al., "Forward-imaging instruments for optical coherence tomography," Opt. Lett. 22(21), 1618-1620 (1997).

8. Y. Pan, H. Xie, and G. K. Fedder, "Endoscopic optical coherence tomography based on a microelectromechanical mirror," Opt. Lett. 26(24), 1966-1968 (2001).

9. L. Huo et al., "Forward-viewing resonant fiber-optic scanning endoscope of appropriate scanning speed for 3D OCT imaging," Opt. Express 18(14), 14375-14384, (2010).

10. W. Drexler and J. G. Fujimoto, Optical Coherence Tomography: Technology and Applications, Springer, Berlin, Heidelberg (2008).

11. H. D. Ford and R. P. Tatam, "Coherent fibre bundles in full-field swept-source OCT," Proc. SPIE 7168, 71682P (2009).

12. T. Xie et al., "Fiber-optic-bundle-based optical coherence tomography," Opt. Lett. 30(14), 1803-1805 (2005).

13. X. Chen, K. L. Reichenbach, and C. Xu, "Experimental and theoretical analysis of core-to-core coupling on fiber bundle imaging," Opt. Express 16(26), 21598-21607 (2008)

14. J. A. Udovich et al., "Spectral background and transmission characteristics of fiber optic imaging bundles," Appl. Opt. 47(25), 4560-4568 (2008).

15. J. H. Han and J. U. Kang, "Effect of multimodal coupling in imaging micro-endoscopic fiber bundle on optical coherence tomography," Appl. Phys. B 106(3), 635-643 (2012).

16. M. D. Risi et al., "Analysis of multimode fiber bundles for endoscopic spectral-domain optical coherence tomography," Appl. Opt. 54(1), 101-113 (2015)

17. H. D. Ford and R. P. Tatam, "Characterization of optical fiber imaging bundles for swept-source optical coherence tomography," Appl. Opt. 50(5), 627-640 (2011).

18. J. H. Han, J. Lee, and J. U. Kang, "Pixelation effect removal from fiber bundle probe based optical coherence tomography imaging," Opt. Express 18(7), 7427-7439 (2010).

19. A. Latrive and A. C. Boccara, "Flexible and rigid endoscopy for highresolution in-depth imaging with full-field OCT," in Biomedical Optics and 3-D Imaging, Miami, Florida (2012). 
20. C. Y. Lee and J. H. Han, "Elimination of honeycomb patterns in fiber bundle imaging by a superimposition method," Opt. Lett. 38(12), 2023-2025 (2013).

21. T. Klein et al., "Megahertz OCT for ultrawide-field retinal imaging with a $1050 \mathrm{~nm}$ Fourier domain mode-locked laser," Opt. Express 19(4), 3044-3062 (2011).

22. B. E. A. Saleh and M. C. Teich, Fundamentals of Photonics, Chapter 9 , Wiley, New York (2007).

23. A. Lukic et al., "Fiber probe for nonlinear imaging applications," J. Biophotonics 9(1-2), 138-143 (2016).
Lara M. Wurster received her BSc and MSc degrees in biomedical engineering from the University of Luebeck, Germany. Currently, she is enrolled as a doctoral student of medical physics at the Center for Medical Physics and Biomedical Engineering at the Medical University of Vienna, Austria. Her research mainly focuses on the design and development of endoscope probes for OCT imaging of internal organs.

Biographies for the other authors are not available. 